\section{Garry Walsh}

Asthmatic and Allergic Inflammation Group, School of Medicine,

University of Aberdeen, UK
The second issue of volume two of Therapeutics and Clinical Risk Management contains a diverse selection of comprehensive review articles and original research papers from leading authors around the world.

Disseminated fungal infection rates are rising as a consequence of the increased potency and use of immunosuppressant agents as therapy in cancer and transplantation; increased numbers of AIDS patients; and growing numbers of patients with additional risk factors for this type of infection. Although newer antifungals such as fluconazole show less toxicity and a greater activity spectrum than do older drugs, several of the preparations in this class demonstrate limited effectiveness, resistance, or erratic oral bioavailability; in addition, mortality from disseminated fungal infection remains at an unacceptable level. Voriconazole, a synthetic derivative of fluconazole, is a member of the second generation of triazoles and our first review, from Pemán and colleagues, considers the efficacy and pharmacokinetics of this broad-spectrum antifungal agent.

It is only relatively recently that the importance of oral mucositis as a side-effect of antitumour therapy has been recognized. This distressing condition not only impacts profoundly on the patient's quality of life but also increases the potential for lifethreatening infections and frequently adversely affects the administration of optimal therapeutic regimens through delayed, decreased or discontinued dosing. Following the publication of internationally recognized guidelines for the management of oral mucositis, D'Hondt and colleagues' timely review summarizes its epidemiology, pathophysiology, and the patient- and therapy-associated risk factors associated with its development. The authors also review recent advances in drug therapy for the prevention and treatment of this potentially devastating condition. The recombinant human keratinocyte growth factor, Palifermin, has proved highly effective in the management of cancer treatment-induced toxicity and the authors provide a thorough overview whilst also emphasizing the importance of non-drug elements of prevention, including systematic oral care and good patient information.

The increase in antibiotic resistant strains of microorganisms is of major concern to healthcare and public health professionals and emphasizes the necessity for the development of new and more effective treatments. Daptomycin, a cyclic lipopeptide antibiotic, has a broad spectrum of activity against both aerobic and anaerobic grampositive organisms and is currently approved for use in skin and soft-tissue infections. Shoemaker and colleagues review its effectiveness in these conditions and consider potential adverse effects and the likelihood of the development of resistance by the target organisms. It appears that daptomycin's unique mechanism of action may allow its use to be extended to the treatment of endocarditis, meningitis, ventriculitis, and peritonitis. The authors provide a useful review of its role in these conditions.

Anemia is a frequent problem during cancer therapy and may be in part a result of tumour-related comorbidities or a side effect of chemo- or radiotherapy. Treatment is reliant either on transfusion or administration of recombinant human erythropoietin. Human erythropoietin therapy has well documented clinical benefits, but frequent dosing is required and response rates can vary between individuals. Wauters and coauthors present a comprehensive review of Darbepoetin alfa, a novel erythropoietic protein with a unique molecular structure, in terms of its clinical development and efficacy. The emphasis on recently published findings including two large, phase III, 
placebo-controlled trials in patients with solid tumors and hematological malignancies; data from these trials demonstrated that Darbepoetin alfa treatment was effective, safe and well-tolerated. The authors also discuss the future potential of optimal iron supplementation strategy and the effect of Darbepoetin alfa therapy on patient survival.

Our next review by Richard Shelton is of great interest as it summarizes the data supporting the introduction in the US of a fixed combination of the atypical antipsychotic olanzapine and the serotonin selective reuptake inhibitor antidepressant fluoxetine (Symbiax ${ }^{\mathrm{TM}}$ ) for the treatment of bipolar depression. This is a controversial area as although fixed combinations of drugs of these classes were once common in neuropsychiatry, their use was discontinued as a majority of patients receiving such a regimen were underdosed for depression against a backdrop of high rates of overdose and suicide. The author concludes that although Symbiax is an important alternative for bipolar depression, uptake by practitioners has been limited due to a number of concerns regarding olanzapine use including weight gain, elevated lipid levels, and increased risk of Type II diabetes.

Higher intraocular pressure (IOP) is considered to be a major risk factor for glaucoma, the second leading cause of blindness world-wide. Robert Noecker has provided a comprehensive review of recent advances in and current approaches to the management of glaucoma and intraocular hypertension. Although glaucoma causes significant morbidity it can be effectively prevented and/or treated in many patients. Recent availability of more powerful ocular hypotensives and an enhanced awareness of the benefits of achieving the lowest IOP possible for each patient have resulted in a significantly more aggressive treatment paradigm. Selection of an ocular hypotensive agent should be based on the primary therapeutic objective of early and aggressive intervention in order to preserve the visual field. The author reviews the clinical efficacy, mechanism of action, safety and tolerability profiles, convenience and compliance of frequently used glaucoma medications.

Rheumatoid arthritis (RA) is a chronic systemic inflammatory disorder. However, while poor disease control leads to joint pain and deformity with consequent functional impairment and loss of independence, patients may also experience significant systemic illness including nodules, vasculitis, pulmonary fibrosis and nerve entrapment. Both RA and extra-articular disease are associated with B cell production of the autoantibody termed rheumatoid factor. Thus there has been much interest in strategies to deplete B cells including the use of the anti-CD20 antibody, rituximab. Our last review by Bryant and Moore provides an overview of the rationale for and experience with rituximab in RA. Several recent studies using rituximab have demonstrated significant declines in RA activity thereby providing evidence for the role of $\mathrm{B}$ cells in RA. The authors conclude that rituximab is a major addition to the increasing therapeutic options for the treatment of patients with RA.

The first original research paper in this issue is from Ständer and colleagues who report interesting findings on the efficacy of the topical calcineurin inhibitors tacrolimus and pimecrolimus in diseases such as prurigo nodularis, generalized and localized pruritus including genitoanal pruritus. Topical calcineurin inhibitors have been shown to rapidly reduce eczematous lesions and give rapid relief of pruritus in atopic dermatitis patients. The results presented in this study suggest that calcineurin inhibitors may be helpful in a subgroup of patients with prurigo nodularis. However, additional larger-scale studies will be required to further evaluate the efficacy of tacrolimus and pimecrolimus in prurigo nodularis.

Our final original research paper has looked at the costs and benefits of influenza vaccination in a group of employees of an Italian District Health Authority. This is an interesting area of research since influenza is the third infectious cause of death in first world countries with significant attendant morbidity. The results presented here suggest that the influenza vaccination strategy was costsaving and that the economic evaluation used in this study might be applicable to other vaccination strategies in other settings. 